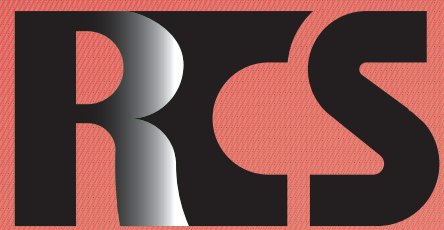

Depósito legal ppi $201502 Z U 4662$

Esta publicación científica en formato digital es continuidad de la revista impresa Depósito Legal: pp $197402 Z$ Z789

- ISSN: 1315-9518 • ISSN-E: 2477-9431

Revista de Ciencias Sociales

Universidad del Zulia. Revista de la Facultad de Ciencias Económicas y Sociales

Vol. XXVII,

No. Especial 3, 2021

Esta publicación científica en formato digital es continuidad de la revista impresa Depósito Legal: pp $197402 Z$ Z789

ISSN: $1315-9518$ 


\title{
Auditoría integral en inventarios y costos de ventas en negocios del Cacao Ecuatoriano*
}

\author{
Erazo Portilla, Carla Mariuxi** \\ Robles Quiñonez, Darío Gustavo**** \\ Cifuentes Quiñonez, Luz Marina***** \\ Saquisari Armijos, Diana Lucia*****
}

\section{Resumen}

La auditoría integral ha progresado en los últimos tiempos, dicho progreso se evidencia en el desarrollo y amplificación de su campo de acción, la presente investigación tiene como objetivo analizar la auditoría integral en inventarios y costo de ventas en negocios del Cacao Ecuatoriano, particularmente se desarrollaron las fases del proceso de auditoría integral desde lo administrativo, y su vinculación con los inventarios y costos de ventas de la empresa. La metodología es de tipo documental-mixta. En este contexto, el procedimiento consistió en ejecutar las fases de auditoría ordenadamente y con una secuencia congruente de actividades, así como aplicación de técnicas como observación, constatación, verificación, conciliación, entrevistas y confirmación, que iniciaron con revisión documental de las actividades. Como resultados se asevera que la auditoría integral pese a brindar informes de aseguramiento, muchas organizaciones no la aplican, destacando la visión innovadora de integrar objetivos y propósitos de éstas para obtener una evaluación global de resultados y desempeño organizacional. Se concluye, que la Auditoría Integral es una evaluación multidisciplinaria, independiente y con visión del sistema, grado y forma de cumplimento de objetivos empresariales, relación con su entorno, y operaciones, haciendo énfasis en su importancia como herramienta gerencial, para toma de decisiones.

Palabras clave: Auditoría integral; inventarios; costos; ventas; negocios.

* Esta investigación es producto del proyecto de investigación titulado: "Calidad de la Gestión Universitaria: Estudio comparativo entre organizaciones ubicadas en las Zonas de Planificación 1 y 4 del Ecuador", financiada por el Vicerrectorado de Investigación, Innovación y Postgrado de la Universidad Técnica "Luis Vargas Torres" de Esmeraldas.

** Magister en Auditoría Integral. Docente en la Universidad Técnica Luis Vargas Torres de Esmeraldas, Ecuador. E-mail: carla.erazo@utelvt.edu.ec; carlitamariuxi@hotmail.com (D) ORCID: https://orcid. org/0000-0001-5974-5523

**** Magister en Auditoría Integral. Docente en la Universidad Técnica Luis Vargas Torres de Esmeraldas, Ecuador. E-mail: dario.robles@utelvt.edu.ec; gdrq11@yahoo.com (iD ORCID: https://orcid.org/00000002-5860-6764

**** Magister en Administración de Empresas. Docente en la Universidad Técnica Luis Vargas Torres de Esmeraldas, Ecuador, E-mail: 1uz.cifuentes@utelvt.edu.ec; lucecitacifuentes@hotmail.com iD ORCID: https://orcid.org/0000-0002-0514-8616

****** Master en Economía y Desarrollo Territorial. Universidad de Huelva, España. Docente en la Universidad Técnica Luis Vargas Torres de Esmeraldas, Ecuador. E-mail: diana.saquisari.armijos@utelvt.edu.ec; diani18_hotmail.com (iD) ORCID: https://orcid.org/0000-0003-4270-9803 


\title{
Comprehensive audit of inventories and sales costs in Ecuadorian Cacao businesses
}

\begin{abstract}
The comprehensive audit has progressed in recent times, such progress is evidenced in the development and amplification of its field of action, the present research aims to analyze the comprehensive audit of inventories and cost of sales in Ecuadorian Cacao businesses, particularly those developed the phases of the integral audit process from the administrative point of view, and its relationship with the inventories and costs of sales of the company. The methodology is documentary-mixed type. In this context, the procedure consisted of executing the audit phases in an orderly fashion and with a consistent sequence of activities, as well as the application of techniques such as observation, verification, verification, conciliation, interviews and confirmation, which began with a documentary review of the activities. As results, it is asserted that the integral audit despite providing assurance reports, many organizations do not apply it, highlighting the innovative vision of integrating objectives and purposes of these to obtain a global evaluation of results and organizational performance. It is concluded that the Comprehensive Audit is a multidisciplinary, independent evaluation with a vision of the system, degree and form of compliance with business objectives, relationship with its environment, and operations, emphasizing its importance as a management tool for decision-making.
\end{abstract}

Keywords: Comprehensive audit; inventories; costs; sales; business.

\section{Introducción}

La auditoría ha progresado en los últimos tiempos, dicho progreso se evidencia en el desarrollo y amplificación de su campo de acción, concentrando nuevas disciplinas que generan una actuación en la vida y desarrollo tanto de entidades públicas como privadas; en este contexto, se determina que la auditoría integral persigue tres objetivos fundamentales: Eficiencia, economía y eficacia. La auditoría integral tiene su nacimiento en el período comprendido de 1980 - 1990 en países como Canadá y Arabia Saudita.

En la realidad ecuatoriana, por su entorno socio económico, no se ha podido determinar el nivel de eficiencia, economía, efectividad y transparencia de los recursos públicos, pues los exámenes de auditoría son aplicados de manera individual y no integral; evitando así una verificación veraz de los elementos de la gestión institucional pública, lo cual evidencia la fundamental importancia de generar un proceso de auditoría integral, que incluya métodos y procedimientos eficaces de control institucional en beneficio de la administración adecuada de recursos públicos, así como privados, generando un ambiente de confianza y credibilidad en todos los aspectos enmarcados en la gestión de recursos de las entidades dentro del marco legal vigente.

La percepción de auditoría integral se cimienta porque las organizaciones y usuarios de la información, así como los auditores que representan la necesidad, se basan en simples revisiones financieras que se realizan en forma periódica por una macro visión que abarca la gestión; finanzas y economía; cumplimiento; $\mathrm{y}$, por supuesto, la evaluación del control interno, que filtre los errores y desviaciones incluso antes de que ocurran o mediante un control preventivo, concurrente y posterior de procesos, así como procedimientos empresariales. En ese sentido, RomeroGarcía, López-Gavira y Pérez-López (2017) sostienen que la "auditoría es necesaria y útil para la economía de cualquier país, puesto que puede aportar un importante valor añadido a la información económico-financiera proporcionada por las empresas" (p.11).

En las empresas, los componentes de costo de ventas e inventarios son uno de los 
más importantes, debido a la complejidad en la identificación y asignación de los costos de ventas con el cargo a las cuentas apropiadas y su valoración exacta, así como al control adecuado de existencias en la recepción, inspección, almacenamiento, valuación y conversión de inventarios en los almacenes o bodegas, generando mayor rotación de los mismos. Realizar una auditoría a estos rubros que se encuentran dentro de las operaciones, es vital para asegurar que los resultados que una compañía presente al final de sus ciclos de operaciones sea razonable.

Esta investigación tiene como objetivo realizar un examen de auditoría integral a la recepción, inspección, almacenamiento, valuación y conversión, al componente de inventario y costo de venta del producto Cacao de la Fundación Maquita Cushunchic Comercializando como Hermanos (MCCH), del 01 de enero a 31 diciembre 2019, así como ejecutar las fases del proceso de auditoría integral al área de inventario de la Fundación Maquita Cushunchic, además opinar sobre la razonabilidad de los saldos relacionados, el sistema de control interno, el cumplimiento de las principales disposiciones legales y la gestión empresarial del área de inventarios de productos y costos de venta, para finalmente, generar el informe de examen integral y las pertinentes acciones correctivas.

En este contexto, la auditoría integral ha pasado a ocupar un lugar importante dentro del ámbito empresarial, como una herramienta de gran importancia para una exitosa administración o gestión, por lo cual se torna imprescindible dominar y ejecutar procesos de auditorías, con la finalidad de establecer fortalezas, así como debilidades que permitan corregir acciones inadecuadas y potenciar el crecimiento de las empresas.

En ese sentido, se realizó una revisión bibliográfica acerca del tema en las principales bases de datos, como son Scopus, Lantidex y SciELO, encontrándose algunos registros, que trataron de alguna manera el rol de las auditorías integrales, los aspectos que incluye un informe de auditoría, así como la gestión de las medianas empresas de América Latina y en Ecuador.

El estudio realizado exigió la utilización de la Investigación documental, para lograr la obtención de información proveniente de documentos oficiales tales como: Estados financieros, con el fin de determinar la razonabilidad de los saldos expuestos; base legal, orientada a establecer el grado de cumplimiento de la normativa aplicable; el plan operativo anual, con la finalidad de verificar el cumplimiento de las metas fijadas, así como el uso de los recursos planificados para su consecución; kardéx de inventarios, liquidación de compras, listado de proveedores, así como manual de funciones y estatutos de la institución, donde se ejecutó la auditoría, que servirá de evidencia para fundamentar el informe.

El manejo inadecuado de los inventarios, al igual que la recepción, inspección, almacenamiento, valuación y conversión del producto en el momento y cantidad incorrecta, incurren siempre en el aumento de costos y disminución de beneficios, necesitando incluso un mayor esfuerzo de parte del personal para obtener una rentabilidad reducida, razón por la cual, este estudio abarca el análisis de la gestión de valuación, conversión, compras e inventario, como herramienta fundamental en el logro de los objetivos institucionales.

Con el desarrollo de esta investigación, se busca conseguir soluciones que proporcionen la gestión de aprovisionamiento de la empresa, lo cual puede traducirse en una reducción importante en los costos de adquisición, compra, almacenaje, transporte y comercialización del cacao, certificando el abastecimiento en el tiempo y lugar requerido por los clientes. Con estos antecedentes, el presente proyecto se justifica toda vez que se pretende establecer a través de la auditoría integral un examen al manejo de los inventarios, para de esta forma perfeccionar la compra, recepción y almacenamiento, con el fin de lograr los objetivos y metas propuestas por la empresa. En ese sentido, el examen de auditoría a los inventarios permite lograr un mejor control de los mismos, aportando eficiencia, eficacia y economía de los recursos 
y materiales para el desarrollo de la gestión institucional.

\section{La auditoría integral: Desde el contexto administrativo}

Con el propósito de ubicar como se ha ido enriqueciendo a través del tiempo la auditoría, es conveniente resolver las contribuciones de los autores que han incidido de manera más significativa a lo largo de la historia de la auditoría empresarial en lo administrativo. Al respecto, Chiriboga (2001) sostiene que:

La auditoría es la revisión y verificación de las cuentas de balance y de pérdidas y ganancias, con el objeto de verificar que las mismas reflejen tanto patrimonio como el resultado de las operaciones de la entidad auditada, de conformidad con los aspectos legales que son aplicables a los principios de contabilidad generalmente aceptados. (p.138)

Por su parte, Aguirre (2005) aclara que el objetivo principal, es la elaboración de un informe dirigido a colocar expresamente "una opinión técnica sobre si dichos estados financieros expresan, en todos los aspectos significativos, la imagen fiel del patrimonio y la situación financiera de la sociedad, así como del resultado de las operaciones en el período examinado" (p.15) conforme a los principios y normas de contabilidad habitualmente aceptados. Asimismo, Barragán y Vergara (2010) sostienen que la auditoria es una revisión sistemática mediante la cual determinará la razonabilidad de los estados financieros dentro de una entidad, con la finalidad de sustentar hechos, así como evidencias encontradas durante la ejecución de actividades. Al respecto, Cedeño-Choéz (2016) manifiesta que:

(...) la auditoria, en su acepción más amplia establece verificar que la información financiera, operacional y administrativa que se presenta es confiable, veraz y oportuna. Es decir, examinar que los hechos, fenómenos $\mathrm{y}$ operaciones se den en la forma como fueron planeados; que las políticas y lineamientos establecidos han sido observados y respetados; que se cumplen con obligaciones fiscales, jurídicas y reglamentarias en general. (p.408)

De igual manera, Taylor y Glezen (2001) manifiesta que: La "auditoría es un examen sistemático de las actividades de una organización en relación con los objetivos específicos, a fin de evaluar el comportamiento, señalar las oportunidades de mejorar y generar recomendaciones para el mejoramiento o para favorecer la acción" (p.145). Sotomayor (2008), al respecto, sostiene que la auditoría administrativa "representa la conjunción de una técnica de control ya existente (la auditoría) y una disciplina que se inicia formalmente y que continúa desarrollándose con nuevos enfoques (la administración)" (p.33). De ahí la existencia de diferentes definiciones en este campo, las cuales enmarcan la asociación de los dos términos y su aplicación en las organizaciones, con base en enfoques que dan relevancia a los procedimientos, ya sea en su operatividad o a lo concerniente a control.

A fin de conseguir una mayor comprensión a cerca de la auditoría integral, resulta significativo hacer hincapié en las conceptualizaciones que la implican. Al respecto, Blanco (2012), sostiene que: $\mathrm{La}$ auditoría integral "es el proceso de obtener y evaluar objetivamente, en un periodo determinado, evidencia relativa a la siguiente temática: la información financiera, la estructura de control interno financiero, el cumplimiento de las leyes y regulaciones pertinentes y la conducción ordenada" (pág.4) hacia el logro de las metas y objetivos que se han propuesto en las empresas; con la finalidad de informar acerca del grado de correspondencia entre estos temas y los indicadores o razonamientos determinados para su evaluación.

De acuerdo a las definiciones anteriores, la auditoría tiene por objeto averiguar la exactitud, integridad y autenticidad de los estados financieros, normativas de control interno, leyes y procedimientos, indicadores de gestión, así como expedientes y demás 
documentos

administrativos-contable

presentados por la dirección, al tiempo de sugerir las mejoras que procedan al respecto.

$\mathrm{Se}$ puede concluir, que las investigaciones en la temática de la auditoria administrativa se encuentran en un bajo nivel de desarrollo, dado el nivel bajo de citas que alcanzan y, además, se evidencia que la mayor parte de los documentos no han sido publicados en revistas indexadas, encontrándose en repositorios institucionales, es decir, se tratan de Tesis de tercer nivel o de documentos internos. La búsqueda en ScienceDirect permitió determinar que es una de las revistas más importante en esta temática, en idioma español. El análisis de los artículos producto de esta revisión consiente recomendar como posibles líneas de investigación: Independencia del auditor y papel de los comités de auditoría, que puedan profundizar en el papel de la Responsabilidad Social Corporativa y el manejo de riesgos, principios que rigen el informe de auditoría y la competencia del auditor.

\subsection{Fases de la auditoría integral: Algunos avances para su concreción}

Los procedimientos analíticos cuando se usan en la planeación de la auditoría, son de gran beneficio para el auditor, con el fin de planear la naturaleza, oportunidad $y$ alcance de los procedimientos, que se usaran en determinadas cuentas (colectivas), así se conocerá el negocio del cliente y sus transacciones, además se identificaran áreas que puedan plantear riesgos más altos. Entre esas fases se encuentra:

a. Planificación especifica: Una vez concluida la planificación preliminar e identificadas las operaciones que requieren de mayor revisión y análisis, para el diseño adecuado del enfoque de la auditoria, será necesario considerar, entre otras las siguientes instrucciones: Aclaración o información adicional, sobre políticas contables aplicadas por la entidad; verificación de la información gerencial importante; procedimientos de diagnóstico, que serán ejecutados; y, obtención de información adicional de algún sistema.

b. Ejecución: En esta fase el auditor debe aplicar los procedimientos establecidos en los programas de auditoría y desarrollar completamente los hallazgos significativos relacionados con las áreas y componentes considerados como críticos, determinando los atributos de condición, criterio, efecto y causa, que motivaron cada desviación o problema identificado. Además, se realiza la "evaluación de los resultados de la gestión y determinación de los hallazgos resultantes para sustentar los comentarios, las conclusiones y recomendaciones que serán incluidas en el informe" (Contraloría General de la República, 2011, p.21). Todos los hallazgos desarrollados por el auditor, estarán sustentados en papeles de trabajo en donde se concreta la evidencia suficiente y competente que respalda la opinión y el informe.

c. Informe: Esta fase comprende también, la redacción y revisión final del informe borrador, el que será elaborado en el transcurso del examen, con el fin de que el último día de trabajo en el campo y previa convocatoria, se comunique los resultados mediante la lectura del borrador del informe a las autoridades y funcionarios responsables de las operaciones examinadas, de conformidad con la ley pertinente.

d. Seguimiento: Esta fase permite verificar el cumplimiento de las recomendaciones que constan en los informes emitidos por auditorias anteriores por parte de las autoridades o funcionarios de la entidad auditada. Al respecto, la Ley Orgánica de la Contraloría General del Estado (Congreso Nacional, 2015) en su artículo 92 indica que:

Las recomendaciones de auditoría, una vez comunicadas a las instituciones del Estado y a sus servidores, deben ser aplicadas de manera inmediata y con el carácter de obligatorio; serán objeto de seguimiento y su inobservancia será sancionada por la Contraloría General del Estado. (p.30)

Por lo expuesto anteriormente, se puede establecer que la auditoría integral involucra un conjunto de fases relacionadas estrechamente, 
que supone el proceso como un solo cuerpo indivisible, en el que sus compendios se separan únicamente para efectos de análisis de sus métodos. Entre los componentes que conforman la auditoría integral se encuentran: Auditoría financiera, de control interno, de gestión y la auditoría de cumplimiento. Al respecto, Blanco (2012) considera a la auditoría financiera, como aquella que proporciona una certeza razonable de los estados financieros, finales o intermedios, tomados de una forma integral, que están libres de manifestaciones erróneas importantes, mientras que la auditoría de control interno, permite evaluar el funcionamiento del sistema de control interno y dictaminar sobre este aspecto.

De igual manera, manifiesta Blanco (2012) que, la auditoría de gestión, evalúa la eficiencia, eficacia y calidad de la gestión empresarial o institucional; en tanto que, la auditoría de cumplimiento, proporciona al auditor una certeza razonable de si las operaciones de la entidad se conducen de acuerdo con las leyes y reglamentos que las rigen. Por lo cual, se considera la auditoria como una herramienta de gran importancia para una exitosa administración empresarial, en la cual se torna imprescindible dominar y ejecutar procesos de auditorías con la finalidad de establecer fortalezas y debilidades que permitan corregir acciones inadecuadas y potenciar el crecimiento de la Fundación Maquita Cushunchic Comercializando como Hermanos (MCCH).

Al respecto, Petit, Piedrahita y Palacio (2016), en su investigación infieren que "promover el equilibrio emocional y cognitivo de la organización desde el liderazgo gerencial durante las auditorias en sistemas de gestión integrados constituye una de las principales estrategias organizacionales para afrontar el tema de la excelencia en gestión organizativa" (p.107).

Para concluir, es importante destacar que la auditoría integral, constituye un proceso novedoso en la gestión óptima de cualquier organización, por lo que requiere ser conceptualizada desde la articulación de los procesos que integran los otros enfoques de auditoría: Por relación de las revisiones financieras, de cumplimiento, control interno y de gestión, a fin de facilitar la construcción de una visión holística del conjunto de responsabilidades que atañen a la gestión empresarial.

En este sentido, se coincide con los planteamientos de Subía-Guerra y TamayoGalarza (2018), quien destaca la visión innovadora que se alcanza al integrar los objetivos y propósitos de las auditorías, con el fin de obtener una evaluación global de los resultados, así como del desempeño de la organización. De igual manera, se está de acuerdo con Báez y Puentes (2018), quienes evidencian en su investigación que la mayoría de las empresas "llevan a cabo una gestión administrativa simplista y cortoplacista, que indica la necesidad de una capacitación para los empresarios (...), en conceptos de administración actualizados que les permita mejorar la gestión de sus organizaciones" (p.81), específicamente en temas de auditoria, que les ayude a tomar decisiones eficaces, así como una mayor sostenibilidad de las empresas en el futuro.

\section{Inventarios y costo de venta: Una conceptualización desde la visión de la auditoría integral}

Considerando la importancia que tienen los inventarios, así como su incidencia en la determinación de los costos de venta en la empresa, como procesos determinantes en los resultados de la auditoría integral, en este apartado se desarrolla de manera breve y sencilla, los aspectos relacionados con la administración del inventario como una herramienta para la optimización de los recursos financieros en una empresa, tomando en cuenta las opiniones al respecto. Durán (2012) manifiesta que:

Los inventarios tienen su origen en los egipcios y demás pueblos de la antigüedad, donde acostumbraban almacenar grandes cantidades de alimentos para ser utilizados en los 
tiempos de sequía o de calamidades. Es así como surge el problema de los inventarios, como una forma de hacer frente a los periodos de escasez. Estos permiten asegurar la subsistencia del negocio y el desarrollo de sus actividades operativas. Esta forma de almacenamiento de todos los bienes y alimentos necesarios para sobrevivir fue lo que motivó la existencia de los inventarios. (p.56)

Los inventarios o stocks, según Díaz (1999), "son la cantidad de bienes que una empresa mantiene en existencia en un momento dado" (p.3), bien sea para la venta ordinaria del negocio o para ser consumidos en la producción de bienes o servicios para su posterior comercialización. De acuerdo con Espinoza (2011), el control de inventarios es una herramienta fundamental en la administración moderna, puesto que esta permite a las empresas y organizaciones conocer las cantidades existentes de productos disponibles para la venta, en un lugar y tiempo determinado, así como las condiciones de almacenamiento aplicables en las industrias.

En ese sentido, se puede decir que el inventario es una relación detallada de los materiales, productos, mercancías que tiene la empresa, almacenados y clasificados según categorías. Según Bustos y Chacón (2012), éstos "aglutinan el conjunto de bienes que las empresas requieren para satisfacer la demanda de los productos que ofertan" (p.241); desde el punto de vista contable, los inventarios están considerados como activos circulantes de suma importancia que afectan directamente a la rentabilidad de la empresa.

De acuerdo a los estándares de información financiera, los inventarios se definen como los activos poseídos para ser vendidos en el curso normal de la operación, en proceso de producción con miras a la venta $o$ en forma de materiales o suministros para ser consumidos en el proceso de producción o en la prestación de servicios; se consideran un activo corriente y en el balance general se relaciona inmediatamente después de las cuentas por cobrar (Duque, Osorio y Agudelo, 2010). Con respecto a la NIC 2, que describe las existencias, González (2009) menciona que "bienes comprados y almacenados para revender (mercaderías); terrenos u otras inversiones inmobiliarias que se tienen para ser vendidas a terceros; productos terminados $o$ en curso y materiales para usarse en el proceso productivo" (p.56).

En ese sentido, Parada (2009) manifiesta que: "Los motivos básicos para crear inventarios son: protegerse contra incertidumbres, permitir la producción y compra bajo condiciones económicamente ventajosas, cubrir cambios anticipados en la demanda y la oferta y mantener el tránsito entre los puntos de producción o almacenamiento" (p.172), convirtiéndose en un valor económico como activo de la empresa.

Por otra parte, el concepto de costo tiene diferentes significados por cuanto está en función de sus estructuras y aplicación. El costo está representado por la suma de los recursos expresados en términos monetarios que se invierten para poder producir un bien o prestar un servicio, que genera beneficios económicos futuros, y se recupera en el momento de la venta de los mismos. El costo, en muchas ocasiones se confunde con el gasto; sin embargo, tiene una diferencia muy específica, se considera costo cuando se realiza la inversión inicial y en el momento en el que se recupera la inversión por medio de la venta del producto, es cuando se convierte en gasto; es decir, que cuando dicha inversión inicial (costo) cumplió con su objetivo, que es generar ingresos a la empresa, entonces se convierte en gasto (López y Cañizares, 2018).

Asimismo, Morales, Smeke y Huerta (2018) manifiestan que el costo se refiere a cualquier erogación o salida de efectivo que realiza la empresa, con el fin de lograr un bien o servicio del cual espera obtener un beneficio en el futuro; en tanto que, un gasto, sería un costo expirado; así como una pérdida, representa un costo ya expirado que nunca generó un ingreso a la empresa; y en ese sentido, un activo, representa un costo sin expirar que se clasifica como tal y se muestra en los estados financieros de la empresa.

Una definición basada en la estructura 
de Materia Prima Directa, Mano de Obra Directa y Costos Indirectos de fabricación, es la de "Edward Menesby, quien sostiene que el costo se define como la medición en términos monetarios, de la cantidad de recursos usados para algún propósito u objetivo, tal como un producto comercial ofrecido para la venta general o un proyecto" (Chambergo, 2014, p.IV-1). Los recursos emplean materia prima, materiales de empaque, horas de mano de obra trabajada, prestaciones, personal salariado de apoyo, suministros y servicios comprados y capital atado en inventario, terrenos, edificios y equipo. Al respecto, sostiene Cárdenas (2016) que en la contabilidad se deben considerar, los tres elementos del costo, la materia prima, el recurso humano que la trabaja, así como todos los costos indirectos del proceso de fabricación.

Ferguson y Gould (1990), definen al costo como un elemento de la actividad económica, así, para el empresario particular esto implica sus obligaciones de hacer pagos en efectivo, en tanto que, para el conjunto de la sociedad, el costo representa los recursos que deben sacrificarse para obtener un determinado bien. Esta definición emplea el término "sacrificios" para referirse a costos que directamente no están relacionados con el dinero en efectivo, como las depreciaciones del activo fijo, pero están involucrados en el proceso productivo, comercial o de servicio.

Harry Howe, define al costo como el precio pagado o la retribución para adquirir un activo. Aplicando a los inventarios, el costo significa en principio la suma de los desembolsos aplicables y cargos incurridos directa o indirectamente al traer un artículo a su condición y localización existente. (Chambergo, 2014, p.IV-2).

Entre las circunstancias que dificultan el tratamiento de los costes indirectos, sobre todo los de gastos generales y de administración, se destacan las siguientes: La propia dimensión o tamaño de la empresa y la dispersión geográfica de sus centros de operaciones y centros administrativos (Aguado, López y Vera, 2013). Es por ello, que Duque, Osorio y Agudelo (2012) señalan que:

\begin{abstract}
La mayoría de las empresas manufactureras necesitan un mayor control presupuestal, una mejor planificación de su producción, un adecuado costeo de sus productos e incluso en muchos casos, buenas políticas de fijación de precios de venta; con el ánimo de en última instancia, generar valor para sus organizaciones. (p.101)
\end{abstract}

Después de revisar y analizar algunos conceptos con relación a inventarios y costo de ventas, se puede concluir que el área de inventarios juega un papel muy importante en las empresas y de manera puntual a las de exportación, es por ello que se debe fortalecer sus controles, es decir, ser cuidadosos con los costos ya sean directos e indirectos, que son imprescindibles en el desarrollo de la productividad empresarial para determinar la utilidad de la empresa y tomar decisiones a través de la optimización de los costos y gastos en el tiempo y momento oportuno, con el fin de minimizar el impacto negativo cuando se trate sobre todo de factores externos que afectan de manera significativa a las empresas.

\section{Auditoría integral en inventarios y costos de ventas en Ecuador}

La globalización y la apertura de los mercados a través de la internacionalización generan en Ecuador mayor perspectiva de crecimiento de las empresas privadas, cuyo desempeño tiene relación directa con los rubros de inventarios y costo de ventas, y están relacionados con los indicadores de eficiencia, eficacia y calidad. Las gerencias en la actualidad deben afrontar nuevos retos empresariales y afianzar la sostenibilidad de los niveles de productividad y rendimiento.

Específicamente, en los negocios comerciales e industriales, el inventario juega un papel fundamental dentro del desarrollo inherente a sus actividades, debido a que es el rubro de donde se obtienen la mayor cantidad de fuentes de efectivo a través de sus ventas, alcanzando liquidez suficiente para solventar 
sus obligaciones inmediatas y sobre todo para operar con normalidad sus procesos instalados (Lopes-Martínez, Gómez-Acosta y AcevedoSuárez, 2012).

Por otra parte, el costo de los inventarios incluirá todos los costos originados de la adquisición y transformación de los mismos, así como otros costos en los que haya incidido para darles su posición y condición en la actualidad (Chávez y Hansen-Holm, 2012); indica de forma específica como se determinan los costos de adquirir mercaderías para ser vendidas a un precio mayor y por consiguiente obtener mayor rentabilidad para el traspaso de bienes o servicios que permita cumplir con el objetivo principal de obtener réditos económicos en sus actividades.

La práctica de auditoría y las operaciones de control en la República del Ecuador, no demuestran la ejecución de auditorías integrales. En las auditorías generadas por empresas privadas, se determina un alto porcentaje de enfoque financiero, y en el sector público, el marco legal y normativo no incluye dentro del sistema de control externo, la auditoría integral, siendo una proporción casi cercano al 95\% de exámenes especiales, cuyo objetivo esencial es auditar el desempeño de las normativas legales.

Entonces, la percepción de auditoría integral se cimenta porque las organizaciones y usuarios de la información, así como los auditores que visualizan la necesidad de desarrollar sus orígenes, basados en simples revisiones financieras que se realizan en forma periódica, necesitan cambiar hacia una macro visión que abarca la gestión, finanzas y economía, cumplimiento, y por supuesto, la evaluación del control interno, que filtre los errores, así como desviaciones, incluso antes de que ocurra o mediante un control previo, concurrente y posterior de procesos y procedimientos. La auditoría integral en los negocios del Ecuador, constituye una oportunidad a los esquemas de control que se encuentran vigentes, puesto que su aceptación en otros países indica la utilidad de un enfoque cabal y holístico de la auditoría.

Al respecto, la Maestría de Auditoría
Integral, desarrollada por la Universidad Técnica Particular de Loja en los últimos cinco años, se presentó como una alternativa de control externo para organizaciones privadas y públicas, fundamentada en que la sinergia generada al reunir los propósitos de cada una de las auditorías: Financiera, de gestión, de cumplimiento legal y de control interno, permitirá obtener sugerencias más eficaces a los gestores y evitar un enfoque parcial sobre aspectos relevantes de la organización. Dar la vuelta a los paradigmas tradicionales de la auditoría y propender a modelos integrales de control ordenados con los aspectos principales de la gestión, que suministren a su vez informes de fortalecimiento que respalden las decisiones gerenciales, constituyen el motivante esencial para el proyecto.

\section{Auditoría integral en inventarios y costos de ventas en la Fundación Maquita Cushunchic}

Los lineamientos de estudio dentro de la auditoría integral, se desarrollaron bajo los criterios de cada una de las auditorías que conforman la auditoría integral, es decir, para la auditoría financiera, se aplicaron las Normas Internacionales de Contabilidad (NIC) No. 1 (Comité de Normas Internacionales de Contabilidad, 2006). Para la auditoría del control interno, que tiene como objetivo la recepción, inspección, almacenamiento, valuación y conversión al componente de inventarios y costo de venta de producto (cacao), es fundamental evaluar el sistema de control interno a través de un modelo de evaluación del control interno aplicable para la empresa.

El cuestionario de control interno se aplicó a la Gestora territorial, Coordinador Comercial, Coordinador agrícola, Contadora Provincial, debido a que son los responsables de los diferentes procesos que se desarrollan en la institución. Las preguntas fueron distribuidas de acuerdo a los componentes del control interno y considerando las funciones que cada uno desempeña. 
La auditoría de cumplimiento, demandó la evaluación del grado de cumplimiento de las principales disposiciones legales a las que se encuentra sujeta la institución como: Constitución de la República del Ecuador, Ley de Economía Popular y Solidaria, Reglamento Interno de Trabajo Maquita Cushunchic Comercializando como Hermanos "MCCH", Reglamento de Aplicación a la Ley de Régimen Tributario Interno, Políticas Generales de MCCH, Planificación estratégica, Manual Productivo Agrícola MCCH, Manual Comercial MCCH, Plan de negociación, Plan Operativo Anual del año 2019 y otras leyes, reglamentos y normas que rigen a las fundaciones.

En lo que respecta a la auditoría de gestión, se orienta a la evaluación de la eficiencia, eficacia y a través del plan operativo anual del año 2013. Bajo este enfoque es necesario señalar en forma clara, la conceptualización de los siguientes criterios: La eficiencia, se relaciona con la optimización en el uso de los recursos; y la eficacia, mide el logro de las metas y objetivos institucionales.

Una vez finalizado el trabajo investigativo, se puede establecer en forma precisa algunos aspectos de mayor relevancia. Que la auditoría de control interno, gestión, financiera y de cumplimiento, permitió al auditor conocer los diferentes procesos de recepción, inspección, almacenamiento, valuación y conversión al componente de inventarios y costo de venta de la Fundación Maquita Cushunchic (MCCH), determinando las debilidades como resultado de la auditoría integral a través de un informe.

Culminado el trabajo de investigación, se manifiesta que se tuvo inconveniente en cierta información, en especial los documentos financieros, por ser confidenciales de la Fundación Maquita Cushunchic MCCH de la ciudad de Esmeraldas, por lo que dificulta al auditor desarrollar su trabajo con normalidad, más cuando la auditoría integral es una evaluación completa a través del conjunto de cuatro auditorías como son: Auditoría de control interno, de gestión, de cumplimiento y financiera.
El realizar la auditoría integral al proceso de recepción, inspección, almacenamiento, valuación y conversión al componente de inventarios y costo de venta de la Fundación Maquita Cushunchic MCCH de la ciudad de Esmeraldas, permitió conocer el estado actual de la Fundación, a través de una evaluación completa del sistema de control interno y las principales acciones correctivas que se deben aplicar en la institución. Por lo tanto, el examen de auditoría integral representó un instrumento importante para entender en la práctica la aplicación de las fases de la auditoría integral producto de la experiencia vivida en el desarrollo de esta auditoría; cumpliendo con el objetivo de colocar en práctica los conocimientos adquiridos en la maestría.

La realidad de la auditoría integral en inventarios y costos de ventas en empresas cacaoteras en Ecuador, se ha convertido en uno de los blancos más importantes en la actualidad para los negocios de exportación, la mayor parte del cacao exportado por Ecuador, ha impulsado a que las empresas exportadoras encuentren en la Auditoría una herramienta que permita generar toma de decisiones eficaces. Aunque la aplicabilidad de la Auditoría integral en este tipo de empresas es muy escasa quizás se encuentran familiarizadas con la auditoría financiera o de control interno, dejando a la auditoría de gestión y de cumplimiento al margen.

Cabe indicar que, de acuerdo a la teoría existente sobre la auditoría integral como su nombre lo indica, la misma permite dar un resultado completo e íntegro a las empresas sin obviar ninguno de los elementos pertinentes para el desarrollo eficiente, eficaz y oportuno para este importante sector económico del país.

Se puede establecer que, los resultados más notables señalan la posibilidad de realizar el examen de auditoría integral en inventarios y costos de ventas en los negocios de Cacao Ecuatoriano, con los beneficios de lograr un enfoque integral y multidisciplinario en las áreas evaluadas; otro aspecto notable, lo constituye la generación de un modelo de 
control que aprovecha de mejor forma los recursos para el logro de los objetivos.

\section{Conclusiones}

En todos los negocios de cacao y más aún los dedicados a las exportaciones se debe aplicar una auditoría integral, para brindar la evaluación y control de los aspectos que integran las instituciones, recursos, desempeño institucional, cumplimiento de normativas, con la finalidad de lograr una visión de la situación actual y corregir posibles irregularidades.

En este sentido, se recomienda la diligencia estricta de las Normas de Control Interno para las entidades, organismos, para obtener mayor control y confianza en las actividades que desempeñan. Asimismo, que el desarrollo de la auditoría integral este a cargo de equipos multidisciplinarios en los varios enfoques que se analizan, para brindar seguridad razonable. La auditoría integral, debe limitar su alcance de manera correcta, puesto que el hecho de presentarse como un examen de amplia cobertura, puede ocasionar insatisfacción de los clientes.

De igual manera, se evidenció que la auditoría interna de la Fundación Maquita Cushunchic MCCH de la ciudad de Esmeraldas de seguimiento a la matriz de recomendaciones emitidas en el informe producto de la auditoría integral, puesto que la misma desde lo administrativo resulta un examen fundamental para la Administración innovadora, debido a que la sinergia existente entre las auditorías que la conforman otorga múltiples beneficios frente a un amplio ámbito de criterios que identifican oportunidades de mejoras en los procesos examinados en un entorno muy cambiante.

De acuerdo a las fases de auditoría integral, se realizó la evaluación de los componentes de inventarios y costo de ventas de la Fundación Maquita Cushunchic Comercializadora como Hermanos, estableciendo la razonabilidad del saldo de las cuentas, el sistema de control interno, el cumplimiento de leyes o disposiciones legales.
En ese sentido, las correctas aplicaciones de las fases de la auditoría en el manejo de los negocios de cacao ecuatoriano, permiten llegar a niveles altos de eficiencia y eficacia, con el fin de otorgar seguridad razonable a las actividades de este sector productivo del país, buscando mayor apertura en los mercados internacionales, lo que generaría la sostenibilidad del empleo y desarrollo económico de Ecuador.

Los negocios del cacao en Ecuador, debido a la creciente inserción en mercados internacionales, conlleva a afrontar nuevos retos empresariales y afianzar la sostenibilidad de los niveles de productividad y rendimiento a través de la aplicación de la auditoría integral, con la finalidad de dar aseguramiento y razonabilidad a sus procesos. Las estrategias que se deben tener en cuenta dentro de los negocios para contar con un óptimo almacenamiento del producto cacao, consisten en asignar un espacio acorde a sus características que permite planificar, organizar y controlar oportunamente los inventarios, con el fin de obtener, así como mejorar significativamente la rentabilidad; de igual manera, se fortalecen los canales de comunicación, logrando diligencia en los procesos a ejecutarse dentro de estos negocios.

Asimismo, es importante fomentar la motivación, el equilibrio tanto emocional como cognitivo de los integrantes de la organización, desde una gestión integral durante las auditorias como estrategia que contribuya al logro exitoso de la misma, alcanzando los objetivos trazados en la empresa. Los resultados se orientan hacia el reconocimiento de la importancia que para una auditoría integral posee la formación y el aprendizaje basado en la autodeterminación, para lo cual es relevante la capacitación de los integrantes de la organización, con el fin de ayudarlos a interiorizar los conceptos estrategicos relacionados con los procesos e indicadores de las auditorias, contribuyendo en una mejor disposición para afrontar la medición y el análisis de cada área funcional y en cada una de las etapas de la auditoria. 
Auditoría integral en inventarios y costos de ventas en negocios del Cacao Ecuatoriano

Erazo Portilla, Carla Mariuxi; Robles Quiñonez, Darío Gustavo;

Cifuentes Quiñonez, Luz Marina y Saquisari Armijos, Diana Lucia

\section{Referencias bibliográficas}

Aguado, J., López, A., y Vera, S. (2013). Repercusiones de la auditoría de costes y preciosen los sistemas de información para la gestiónempresarial: el caso de Indra Sistemas. Revista de Ciencias Sociales (Ve), XIX(2), 402-414.

Aguirre, J. M. (2005). Auditoría y control interno. Editorial Grupo Cultural.

Báez, M. D. P., y Puentes, G. A. (2018). Parámetros financieros para la toma de decisiones en pequeñas y medianas empresas del municipio Duitama, Colombia. Revista de Ciencias Sociales (Ve), XXIV(1), 67-84.

Barragán, C., y Vergara, C. (2010). Responsabilidad del control interno frente al fraude (Tesis de postgrado). Universidad Militar Nueva Granada, Bogotá, Colombia.

Blanco, Y. (2012). Auditoría integral: Normas y procedimientos. Ecoe Ediciones.

Bustos, C. E., y Chacón, G. B. (2012). Modelos determinísticos de inventarios para demanda independiente: Un estudio en Venezuela. Contaduría y Administración, 57(3), 239-258.

Cárdenas, R. A. (2016). Costos 1. Instituto Mexicano de Contadores Públicos, A. C..

Cedeño-Choéz, P. J. (2016). Auditoría integral a los activos fijos depreciables. Dominio de las Ciencias, 2(3), 406417.

Chambergo, I. (2014). Análisis de la importancia del estado de costo de producción y venta y el estado de resultados en las Normas Internacionales de Información Financiera - NIIF/IFRS. Actualidad Empresarial, (301), IV-1-IV-14.

Chávez, L. A., y Hansen-Holm, M. A. (2012).
NIIF para las Pymes: Teoría y práctica. Hansen Holm \& Co.

Chiriboga, A. (2001). La auditoría administrativa. Vallesco, S.A.

Comité de Normas Internacionales de Contabilidad (2006). Normas Internacionales de Contabilidad No.1: Presentación de Estados Financieros. https://www.nicniif.org/files/NIC\%20 $1 \% 20$ Presentacion $\% 20 \mathrm{de} \% 20$ Estados\%20Financieros.pdf

Congreso Nacional (2015). Ley Orgánica de la Contraloría General del Estado. Registro Oficial Suplemento 595 de 12-jun.-2002. Última modificación: 18-dic.-2015. https://www.oas.org/ juridico/PDFs/mesicic5 ecu ane cge 23 ley_org_cge.pdf

Contraloría General del Estado (2011). Guía Metodológica para la Auditoría de Gestión, Acuerdo No. 047 CG, de 22 de diciembre. https://www.studocu.com/ ec/document/universidad-tecnica-demanabi/laboratorio-de-auditoria-degestion-pp/acuerdo-047-cg-2011-gu iametodoligicaparaauditoriadegesti on/12143598

Díaz, A. (1999). Gerencia de inventarios. Ediciones IESA.

Duque, M. I., Osorio, J. A., y Agudelo, D. M. (2010). Los inventarios en las empresas manufactureras, su tratamiento y su valoración. Una mirada desde la contabilidad de costos. Contaduría Universidad de Antioquia, (56), 61-79.

Duque, M. I., Osorio, J. A, y Agudelo, D. M. (2012). Analisis de la aplicacion del sistema de costeo estándar en las empresas manufactureras colombianas. Revista del Instituto Internacional de Costos, (E), 97-123.

Durán, Y. (2012). Administración del inventario: Elemento clave para la optimización de las utilidades en las empresas. Visión Gerencial, (1), 55- 
78.

Espinoza, O. (2011). La administración eficiente de los inventarios. Editorial La Ensenada.

Ferguson, C., y Gould, J. (1990). Teoría microeconómica. Fondo de Cultura Económica.

Gutiérrez, C. (2009). Las existencias. Pecvnia: Revista de la Facultad de Ciencias Económicas y Empresariales, Universidad de León, 0(2009), 5576. http://dx.doi.org/10.18002/pec. v0i2009.775

Lopes-Martínez, I., Gómez-Acosta, M. I., y Acevedo-Suárez, J. A. (2012). Situación de la gestión de inventarios en Cuba. Ingeniería Industrial, XXXIII(3), 317-330.

López, A. A., y Cañizares, M. (2018). El control interno en el sector publico ecuatoriano: Caso de Estudio: Gobiernos autónomos descentralizados cantonales de Morona Santiago. Cofin Habana, 12(2), 51-72.

Morales, P. B., Smeke, J., y Huerta, L. (2018). Contabilidad: Costos gerenciales. Instituto Mexicano de Contadores Públicos.
Parada, O. (2009). Un enfoque multicriterio para la toma de decisiones en la gestión de inventarios. Cuadernos de Administración, 22(38), 169-187.

Petit, E. E., Piedrahita, G. A., y Palacio, A. A. (2016). Estrategia organizacional para afrontar auditorias en sistemas de gestión integrados. Revista de Ciencias Sociales (Ve), XXII(2), 92-110.

Romero-García, J. E., López-Gavira, M. R., y Pérez-López, J. A. (2017). Servicios adicionales de auditoría. Independencia del auditor y permisividad de la legislación. Revista de Ciencias Sociales (Ve), XXIII(4), 9-22.

Sotomayor, A. A. (2008). Auditoria administrativa: Proceso y aplicación. McGraw-Hill Interamericana Editores S. A.

Subía-Guerra, J. C., y Tamayo-Galarza, G. N. (2018). Experiencias de auditoría integral en el Ecuador. VinculaTégica Efan, 67-72. http://www.web. facpya.uanl.mx/Vinculategica/ vinculat $\%$ C 3\%A9gica $2 / 9 \% 20$ SUBIDA TAMAYO.pdf

Taylor, D. H., y Glezen, W. (2001). Auditoría empresarial. Ctheroung Vaegoss. 